\title{
Evidence for the Role of MAP1B in Axon Formation
}

\section{Christian Gonzalez-Billault, ${ }^{*}$ Jesus Avila, ${ }^{*+}$ and Alfredo Cáceres*+}

\author{
${ }^{*}$ Centro de Biologia Molecular, Consejo Superior de Investigaciones Cientificas, Universidad

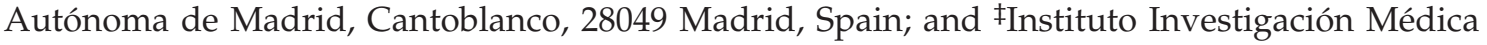 \\ Mercedes y Martín Ferreyra, (INIMEC-Consejo Nacional de Investigaciones Cientificas y Técnicas de \\ Argentina), Córdoba, Argentina
}

Submitted December 14, 2000; Revised March 30, 2001; Accepted April 30, 2001

Monitoring Editor: David Drubin

\begin{abstract}
Cultured neurons obtained from a hypomorphous MAP1B mutant mouse line display a selective and significant inhibition of axon formation that reflects a delay in axon outgrowth and a reduced rate of elongation. This phenomenon is paralleled by decreased microtubule formation and dynamics, which is dramatic at the distal axonal segment, as well as in growth cones, where the more recently assembled microtubule polymer normally predominates. These neurons also have aberrant growth cone formation and increased actin-based protrusive activity. Taken together, this study provides direct evidence showing that by promoting microtubule dynamics and regulating cytoskeletal organization MAP1B has a crucial role in axon formation.
\end{abstract}

\section{INTRODUCTION}

Neurons are highly polarized cells that contain a single long axon and multiple dendrites. Polarization occurs when one of the multiple neurites emerging from the cell body initiates a phase of rapid elongation, becoming the axon; the remaining neurites will develop as dendrites (Bradke and Dotti, 1997, 1999; Dotti et al., 1998). Because microtubule assembly and stabilization play an essential role in axon formation (Mitchison and Kirschner, 1989) a great deal of attention has been devoted to identify factors controlling microtubule organization and dynamics in nerve cells. Current evidence favors the view that several of the distinctive properties of neuronal microtubules, such as increased stability and spatial differentiation, arise from the developmentally regulated expression of structural microtubule-associated proteins (MAPs), which are notably abundant in neurons (Maccioni and Cambiazo, 1995). Therefore, it is likely that the expression of these proteins along neuronal development may affect neuronal polarization.

MAP1B (Bloom et al., 1995) is the first MAP that is specifically expressed during neural development and that is especially prominent in neurons that are actively extending axons (Calvert and Anderton, 1985; Garner et al., 1990; Fischer and Romano-Clarke, 1991; Ulloa et al., 1993; Black et al., 1994: DiTella et al., 1996; Gordon-Weeks and Fischer, 2000). These findings led to the hypothesis that MAP1B might be involved in axon formation by regulating microtubule dynamics. Evidence in favor of this proposal came from antisense experiments showing that MAP1B suppression reduces laminin-promoted axonal elongation (DiTella et al., 1996) and neurite outgrowth in PC12 cells (Brugg et al.,

\footnotetext{
‡ Corresponding author. E-mail address: acaceres@immf.uncor.edu or javila@cbm.uam.es.
}

1993). More recently, it was shown that in Drosophila, an MAP1B-like protein is required for proper axonal and dendritic development (Hummel et al., 2000; Roos et al., 2000), and that microscale chromophore-assisted laser inactivation of phosphorylated MAP1B altered growth cone turning behavior in cultured neurons (Mack et al., 2000). The generation of MAP1B mutant mice has also provided additional important information. Despite discrepancies concerning the severity of the effects, all studies demonstrate that MAP1B-deficient mice have an impairment of brain development (Edelman et al., 1996; Takei et al., 1997; GonzalezBillault et al., 2000; Meixner et al., 2000). Recently, mice with disrupted tau and MAP1B genes have been generated; the phenotype of these animals is markedly more severe than the one of single-tau or MAP1B mutant mice, suggesting some cooperative functions of these MAPs during brain development. (Takei et al., 2000)

Unfortunately, none of these studies examined the consequences of MAP1B gene suppression on cytoskeletal organization, and therefore the functional involvement of $\mathrm{MAP} 1 \mathrm{~B}$ as a key regulator of microtubule dynamics during neuronal polarization has remained largely unexplored. In the present study, we have addressed this issue by examining the morphology, cytoskeletal organization, and dynamics of microtubule assembly in cultured hippocampal pyramidal neurons from a hypomorphous MAP1B mutant mouse line (Gonzalez-Billault et al., 2000) obtained by a gene-trapping approach (Chowdhury et al., 1997).

\section{MATERIALS AND METHODS}

\section{Generation and Genotyping of MAP1B Mutant Mice}

The generation of heterozygous mice lacking a copy of MAP1B by a gene trap approach has been described elsewhere (Chowdhury et 
al., 1997; Gonzalez-Billault et al., 2000). The gene-trapping vector (IRES $\beta$ geo) contained the EN-2 splice acceptor sequence, and IRES sequence, the neo gene, the lac $Z$ gene, and the simian virus 40 polyadenylation signal. To genotype MAP1B mutant mice, genomic DNA from mouse tails were isolated and analyzed by polymerase chain reaction with oligonucleotides corresponding to the neo sequence (Chowdhury et al., 1997).

\section{Protein Extracts and Western Blots}

Protein extracts were prepared from the embryonic spinal cord in 20 mM HEPES pH 7.4 containing $0.1 \mathrm{M} \mathrm{NaCl}, 10 \mathrm{mM} \mathrm{NaF}, 1 \mathrm{mM}$ $\mathrm{Na}_{3} \mathrm{VO}_{4}, 5 \mathrm{mM}$ EDTA, $1 \mu \mathrm{M}$ okadaic acid, and protease inhibitors (2 $\mathrm{mM}$ phenylmethylsulfonyl fluoride and $10 \mu \mathrm{g} / \mathrm{ml}$ aprotinin, leupeptin, and pepstatin). Protein samples were separated in SDSPAGE, electroblotted onto nitrocellulose sheets, developed with an ECL chemiluminescence system, and quantified in a Molecular Dynamics densitometer.

\section{Cell Culture}

For the preparation of cultures, E18-19 embryos were removed aseptically from pregnant mice and placed in sterile Petri dishes. Homozygous MAP1B-deficient embryos were distinguished from controls by their abnormal limb posture (Gonzalez-Billault et al., 2000). Dissociated cultures of hippocampal pyramidal cells from embryonic mice brain tissue were then prepared as described previously (Cáceres et al., 1986; Paglini et al., 1998). Cultures were performed with the hippocampi of E18-19 embryos because at this time point hippocampal pyramidal neurons that develop in situ are beginning to extend neurites. To bind laminin to the substrate, polylysine-coated coverslips were soaked in Neurobasal medium containing mouse EHS laminin at a concentration of $20 \mu \mathrm{g} / \mathrm{ml}$ overnight at $4^{\circ} \mathrm{C}$ as described (DiTella et al., 1996). For some experiments nocodazole $(5 \mu \mathrm{g} / \mathrm{ml})$ was added to the culture medium, and cells were incubated in the presence of the microtubule-depolymerizing agent for different time periods.

\section{Primary Antibodies}

The following primary antibodies were used in this study: a monoclonal $(\mathrm{mAb})$ against tyrosinated $\alpha$-tubulin (clone TUB-1A2; Sigma, St. Louis, MO) diluted 1/200; a mAb against MAP1B (clone 125; Gonzalez-Billault et al., 2000) diluted 1/150; a rabbit polyclonal antibody against MAP2 diluted 1/2000 (Sanchez-Martin et al., 1998); a mAb against tau (clone tau-1; Cáceres et al., 1992) diluted 1/100; a rabbit polyclonal antibody against detyrosinated $\alpha$-tubulin diluted 1/250 (Cáceres et al., 1992); and the rabbit polyclonal antibody 196 against $\beta$ II-tubulin diluted 1/100 (Armas-Portela et al., 1999).

\section{Immunofluorescence}

Cells were prepared for immunofluorescence with the use of two procedures: 1) Fixation for 20 min with warmed $4 \%$ paraformaldehyde- $0.12 \mathrm{M}$ sucrose in phosphate buffer, $\mathrm{pH} 7.2$; and 2) extraction with detergent to prepare "cytoskeletons fractions" according to the procedure of Brown et al. (1992). For this procedure, the cells were washed for $30 \mathrm{~s}$ with buffer PHEM (60 mM piperazine- $N, N^{\prime}$-bis(2ethanesulfonic) acid, $25 \mathrm{mM}$ HEPES, $10 \mathrm{mM}$ EGTA, $2 \mathrm{mM} \mathrm{MgCl}{ }_{2}$, $\mathrm{pH}$ 6.9) followed by extraction ( $2 \mathrm{~min}$ ) with $0.2 \%$ saponin in buffer PHEM containing $10 \mu \mathrm{M}$ taxol. Biochemical (Cáceres et al., 1992; DiTella et al., 1996) and immunofluorescence (Brown et al., 1992) studies have established that this procedure selectively removes soluble tubulin, and that the cytoskeletal fraction contains polymerized tubulin. Quantitative Western blotting has also revealed that this fraction reproducibly represents between 20 and $25 \%$ of the total cellular protein of unextracted cells, which is almost the same value obtained by other studies with the use of slightly different extraction procedures (Drubin et al., 1985; Ferreira et al., 1989; Ferreira and Cáceres, 1989). After extraction the cells were fixed for 20 min with warmed $2 \%$ paraformaldehyde- $0.05 \%$ glutaraldehyde in buffer PHEM, pH 6.9. Cultures were processed for immunofluorescence as described. For some experiments rhodamine-phalloidin (Molecular Probes, Eugene, OR) was included with the secondary antibody to visualize F-actin. The cells were analyzed with a confocal scanning microscope or with an inverted microscope (Carl Zeiss Axiovert $35 \mathrm{M}$ ) equipped with epifluorescence. The relative intensities of tubulin, MAP1B, MAP2, and tau immunofluorescence were evaluated in fixed unextracted cells or in detergent-extracted cytoskeletons with the use of quantitative fluorescence techniques. To image labeled cells, the incoming epifluorescence illumination was attenuated with glass neutral density filters. Images were formed on the faceplate of a Silicon Intensified Target camera, set for manual sensitivity, gain and black level (Paglini et al., 1998a,b). Images were digitized directly into a Metamorph/Metafluor image processor controlled by a host PC computer (Universal Imaging, West Chester, PA). Fluorescence intensity measurements were perfumed within the cell body and neurites of identified neurons; with the use of these data, we then calculated the average fluorescence intensity within the cell body, and inner, middle, and distal third of identified neurites. Background levels were those detected in unlabeled cells. Photographs were printed with the use of Adobe Photoshop.

\section{Morphometric Analysis}

To measure neurite length and growth cone shape parameters (growth cone area, number, and length of filopodia) antibody- or phalloidin-labeled cells were randomly selected and traced from a video screen with the use of the morphometric menu of the Metamorph (Paglini et al., 1998a,b). The following neuritic shape parameters were evaluated: total axonal length, length of minor processes, and total neuritic length (the sum of axonal length plus one of the minor processes). A total of 300 cells belonging to three different low-density cultures were analyzed for each experimental condition and time point. Differences among groups were analyzed by the use of analysis of variance and Student-Newman-Keuls test.

\section{RESULTS}

\section{Delayed Axonal Formation and Growth Cone Abnormalities in Cultured Neurons from MAP1B Mutant Mice}

MAP1B mutant mice were generated with the use of a gene trapping vector (IRES $\beta$ geo) transfected into R1 ES cells (Chowdhury et al., 1997; Figure 1A). The insertion of the vector into the MAP1B locus results in premature termination of MAP1B translation and the expression of a fusion protein with $\beta$-galactosidase and neomycin resistance activities, under the control of the MAP1B promoter (Chowdhury et al., 1997). Mice carrying this mutation express trace amounts of MAP1B, display severe cortical lamination defects, and die shortly after birth (Gonzalez-Billault et al., 2000). In this study the genotype of the embryos used for the cell culture experiments was confirmed through Western blot analysis of spinal cord extracts reacted with monoclonal antibodies against MAP1B (clone 125; Gonzalez-Billault et al., 2000) and $\beta$-galactosidase (Figure 1B).

Cultured hippocampal pyramidal neurons have been extensively used to study axon formation, as well as the expression of MAPs during neuronal morphogenesis (Craig and Banker, 1994). Many of these studies have used laminin as an adhesive substrate, a molecule capable of enhancing polarization by promoting axonal elongation (Lein et al., 1992; Lochter and Schachner, 1993; DiTella et al., 1996). 
A

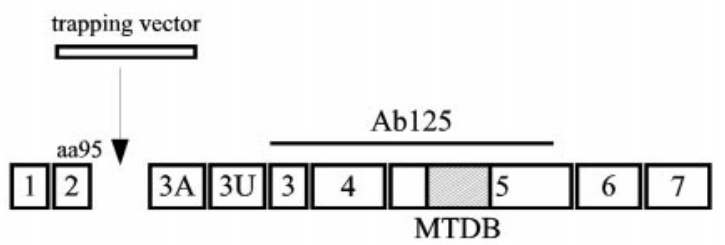

B

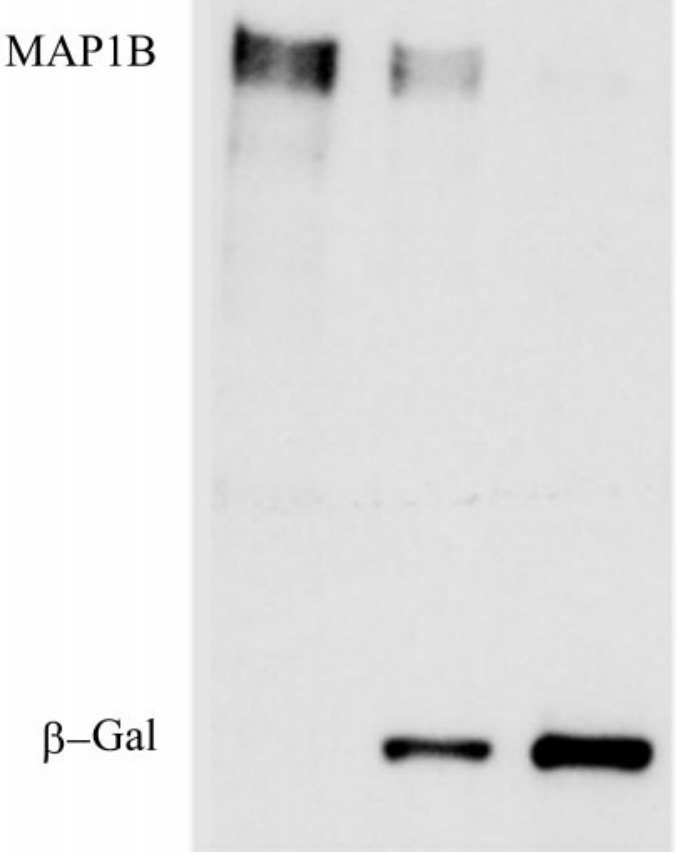

Figure 1. (A) Structure of MAP1B gene. Seven coding exons (1-7) and two noncoding ones ( $3 \mathrm{~A}$ and $3 \mathrm{U}$ ) of the MAP1B protein are shown. The trapping vector insertion occurs immediately after exon 2, which encodes the N-terminal 95 amino acids of the protein. The epitope of antibody 125 used to confirm the genotype of animals is depicted as a straight line. The hatched box represents the microtubule-binding domain (MTBD). (B) Western blot analysis of spinal cord extracts from wild-type $(+/+)$, heterozygous $(+/-)$, and homozygous $(-/-)$ MAP1B mutant mice reacted with a $\mathrm{mAb}$ against MAP1B and $\beta$-galactosidase ( $\beta$-Gal). Note that the extracts obtained from the homozygous $(-/-)$ MAP1B contain trace amounts of MAP1B and high levels of $\beta$-Gal. Ten micrograms of protein was loaded in each lane.

Therefore, to assess the consequences of the disruption of the MAP1B gene on neuronal polarization, we first examined hippocampal pyramidal neurons cultured on laminin for $2 \mathrm{~d}$. At this time point, virtually all neurons from wildtype (WT) animals have become polarized, displaying a long and thin axon and several (3-5) much shorter processes or minor neurites (Figure 2A). Axon-like neurites were also detected in neurons (55\%) from MAP1B-deficient mice; however, these processes were several times shorter than equiv- alent ones from WT animals (Figure 2B). The remaining neurons lack axons, but display minor processes that appear to have an equivalent length to those of control cells. To test whether this phenomenon reflected a retraction of previously formed axons or an impairment in axon development, the neurite outgrowth response of cultured neurons from WT and mutant animals was quantitated at different time points after plating. For this analysis, an axon-like neurite was defined as a process at least twice as long as any other neurite of the same cell, and with a minimum length of 50 $\mu \mathrm{m}$ (Dotti et al., 1998; Cáceres and Kosik, 1990). The results obtained clearly revealed that neurons from MAP1B mutant mice have a selective and significant inhibition of axon formation, a phenomenon that is likely to reflect a delay in axon outgrowth and also a reduced rate of elongation (Figure $2, \mathrm{C}-\mathrm{F}$ ).

To further characterize the morphology of MAP1B-deficient neurons high-resolution confocal microscopy was used. This analysis revealed two additional important alterations: 1) An increase in actin-based protrusive activity resulting in the appearance of numerous short filopodial extensions and growth cone-like structures around the cell body and along neurites (Figure 3, A-C); and 2) A significant change in the shape of axonal growth cones, characterized by a retraction of the growth cone lamellipodial veil and a decrease in the number of radial striations (a chevron arrangement of actin ribs) that extends from the central growth cone region toward the periphery (Figure 3, D-G, and Table 1).

\section{Microtubule Assembly and Dynamics Are Altered in Axons of MAP1B-deficient Neurons}

To determine whether MAP1B affects microtubule assembly and/or stability in developing neurons, we first addressed whether axons formed without MAP1B differ from control ones in their content of microtubule polymer. For this experiment, cultures were fixed after detergent extraction performed under microtubule-stabilizing conditions and processed for immunofluorescence with antibodies against tubulin. This method removes unassembled tubulin from the cell, so that the tubulin staining remaining in such cells is attributable to microtubules (Brown et al., 1992; see MATERIALS AND METHODS). $\beta$-Tubulin fluorescence intensity in "cytoskeletal preparations" was used as a relative measure of polymer mass because previous studies have demonstrated that it is present at a constant stoichiometry in microtubules (Brown et al., 1992); besides, the specific antibody to $\beta$-tubulin (Gonzalez-Billault et al., 2000) that we used stains all microtubules uniformly within the cell body, axons, and minor processes. Quantitative measurements revealed that the intensity of microtubule staining within axons, but not minor neurites, was significantly lower in MAP1B-deficient neurons than in WT ones (Figure 4, A and B). Because no decrease in $\beta$-tubulin immunofluorescence was detected when measurements were performed in cells fixed before detergent extraction (Figure 4C), our observations suggest that MAP1B depletion is altering microtubule formation, by decreasing assembly and/or stability.

To distinguish between these possibilities we examined the relative amounts of two posttranslationally modified tubulins in axonal microtubules of control and MAP1Bdeficient neurons. Several studies have shown that the rel- 
C. Gonzalez-Billault et al.
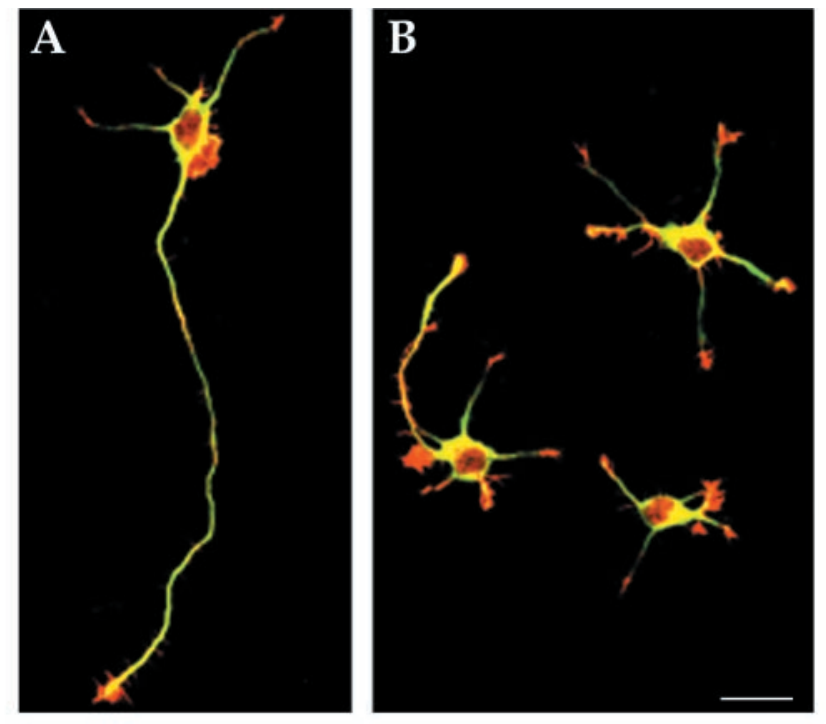

C
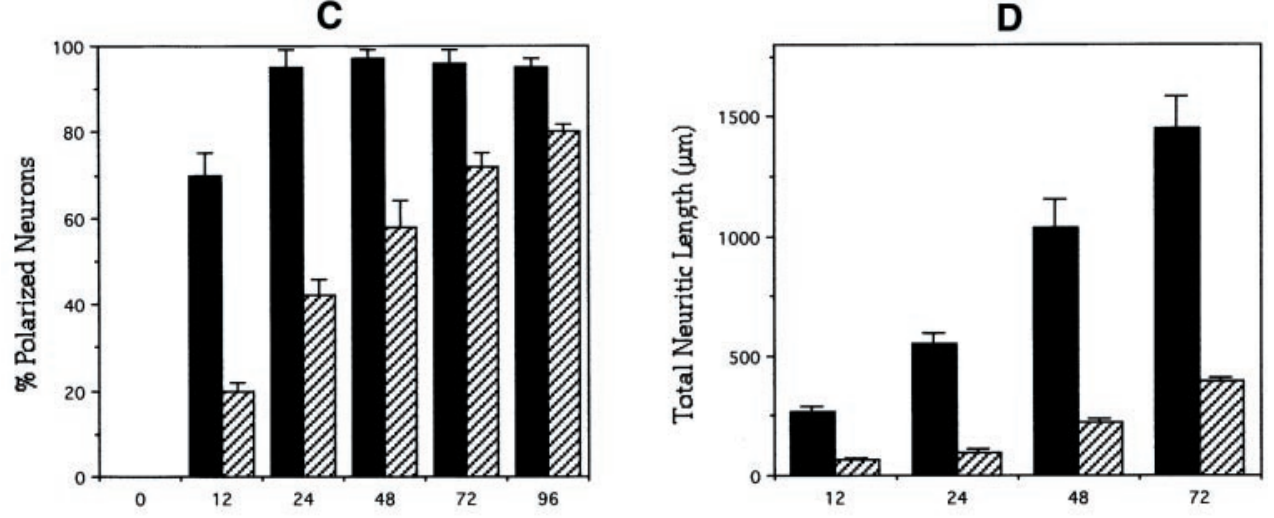

E

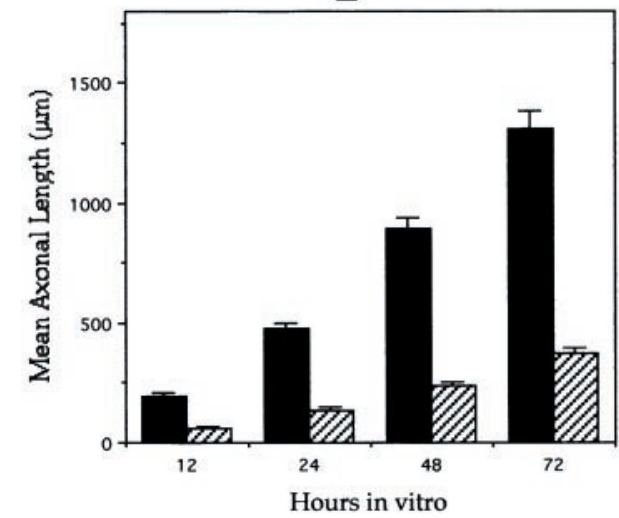

$\mathbf{F}$

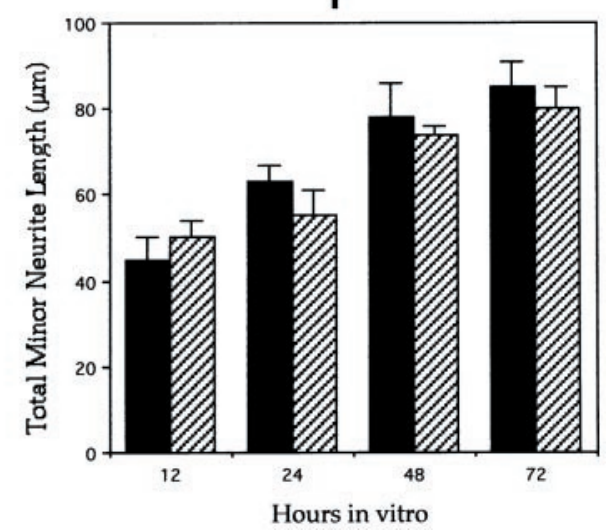

Figure 2. General morphology and morphometric parameters of control and MAP1B-deficient neurons. (A) Confocal micrograph showing a polarized hippocampal pyramidal neuron from a wild-type animal. The cell that displays one single long axon and several much shorter minor neurites was maintained in culture for $2 \mathrm{~d}$. The cell is double labeled with a mAb against tyrosinated $\alpha$-tubulin (green) and rhodamine-phalloidin (red). (B) Confocal micrograph showing cultured hippocampal pyramidal neurons from a homozygous (-/-) MAP1B mutant mice. The cells were cultured for $2 \mathrm{~d}$ and stained as in B. Note that only one cell is polarized and displays a very short axon-like neurite. Bar, $20 \mu \mathrm{m}$. (C) Graph showing the percentage of cells displaying axon-like neurites in cultures from wild-type ( $\square$ ) and MAP1B-deficient (שיC) mice. (D-F) Graphs showing changes in total neuritic length (D), axonal length (E), and total minor neuritic length (F) in hippocampal cell cultures from wild-type ( $\square$ ) and MAP1B-deficient (ש/ ) mice. Note the significant and selective decrease of axonal length in the MAP1B-deficient neurons. Values represent the mean \pm SEM. 

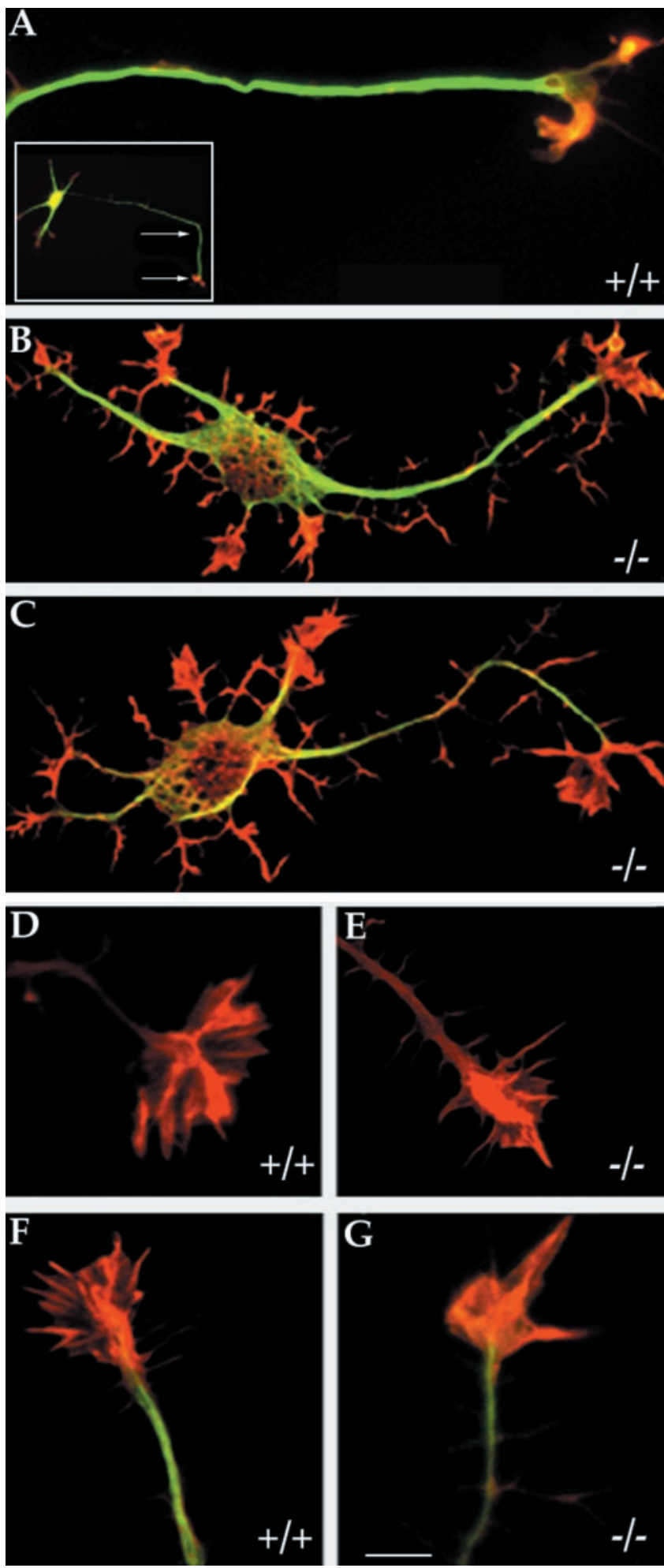

Figure 3. High-resolution analysis of control and MAP1B-deficient neurons. (A) High-resolution confocal micrograph showing the distal axonal end of a hippocampal pyramidal neuron from a WT mouse. A low-power micrograph of the neuron is shown in the inset; the arrows indicate the distal axonal segment. (B and C)
Table 1. Growth cone shape parameters

\begin{tabular}{lccc}
\hline & \multicolumn{2}{c}{ Growth cone area } & \begin{tabular}{c} 
Number of \\
\cline { 2 - 3 }
\end{tabular} \\
\cline { 2 - 3 } & Axons & $\begin{array}{c}\text { Minor } \\
\text { neurites }\end{array}$ & $\begin{array}{c}\text { mislocalized } \\
\text { growth cones }\end{array}$ \\
\hline WT neurons & $180 \pm 22$ & $65 \pm 10$ & $0.20 \pm 0.05$ \\
MAP1B-deficient neurons & $88 \pm 15^{\mathrm{a}}$ & $72 \pm 8$ & $5.5 \pm 0.5^{\mathrm{a}}$ \\
\hline
\end{tabular}

Each value represents the mean \pm SEM. Area values are expressed in square micrometers. Growth cones were considered mislocalized where they were located around the cell body or along neuritic shafts of either minor processes or axons. All measurements were performed in neurons that have been in culture for $48 \mathrm{~h}$. A total of 200 growth cones was analyzed for each experimental group.

a Values significantly different from those of WT neurons.

ative abundance of tyrosinated (tyr) and detyrosinated (detyr) $\alpha$-tubulin in microtubules correlates with its stability properties such that tyr-tubulin is especially enriched in the more dynamic microtubule polymer, whereas detyr-tubulin is enriched in the more stable one (Brown et al., 1992; Baas et al., 1993; Li and Black, 1996). Thus, the distribution and relative levels of these two posttranslational modifications of $\alpha$-tubulin can be used as an indirect, but reliable, assay of microtubule dynamics. This correlation is particularly strong in growing axons, in which the more dynamic polymer is highly concentrated at neuritic tips, whereas the more long-lived polymer is prominent in the proximal axon (Figure $5, \mathrm{~A}-\mathrm{C}$ ). This pattern is clearly altered in axons from MAP1B-deficient neurons; thus, a dramatic decrease in tyrmicrotubule staining paralleled by an increase in detyrmicrotubule labeling is evident at the distal axonal segment (Figure 5, D-F). Quantitative measurements confirmed these observations, and clearly revealed that the balance between dynamic and stable microtubule polymer is significantly altered in axons from MAP1B-deficient neurons (Figure 5, G and $\mathrm{H})$.

To complement these experiments we examined whether MAP1B mutation alters microtubule sensitivity to depolymerizing agents. For such a purpose, the effect of treatment with nocodazole on the loss of tubulin polymer from the distal part of the axon contiguous to the growth cone was measured. The polymer in this region is labile to treatment

High-resolution confocal micrographs showing the morphology of cultured hippocampal pyramidal neurons from MAP1B-deficient mice. The cells $(\mathrm{A}-\mathrm{C})$ were double labeled with a mAb against tyrosinated $\alpha$-tubulin (green) and rhodamine-phalloidin (red). Note that MAP1B-deficient neurons display numerous short filopodial extensions and growth cone-like structures around the cell body and along neurites. Bar, $5 \mu \mathrm{m}$. (D and E) High-power confocal micrographs showing the morphology of the actin cytoskeleton of axonal growth cones from wild-type $(+/+)$ and MAP1B-deficient $(-/-)$ neurons as reveled by staining with rhodamine-phalloidin. ( $F$ and G) Equivalent images to those shown previously but also stained with a mAb against tyrosinated $\alpha$-tubulin (green). Note the reduction in the size of the growth cone lamellipodial veil and the decrease in the number of radial striations in the mutant neurons. Bar, $5 \mu \mathrm{m}$. 


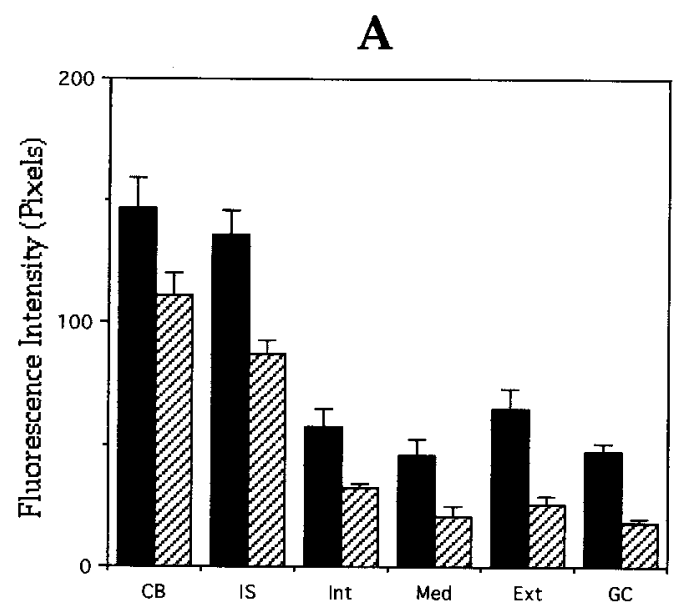

B
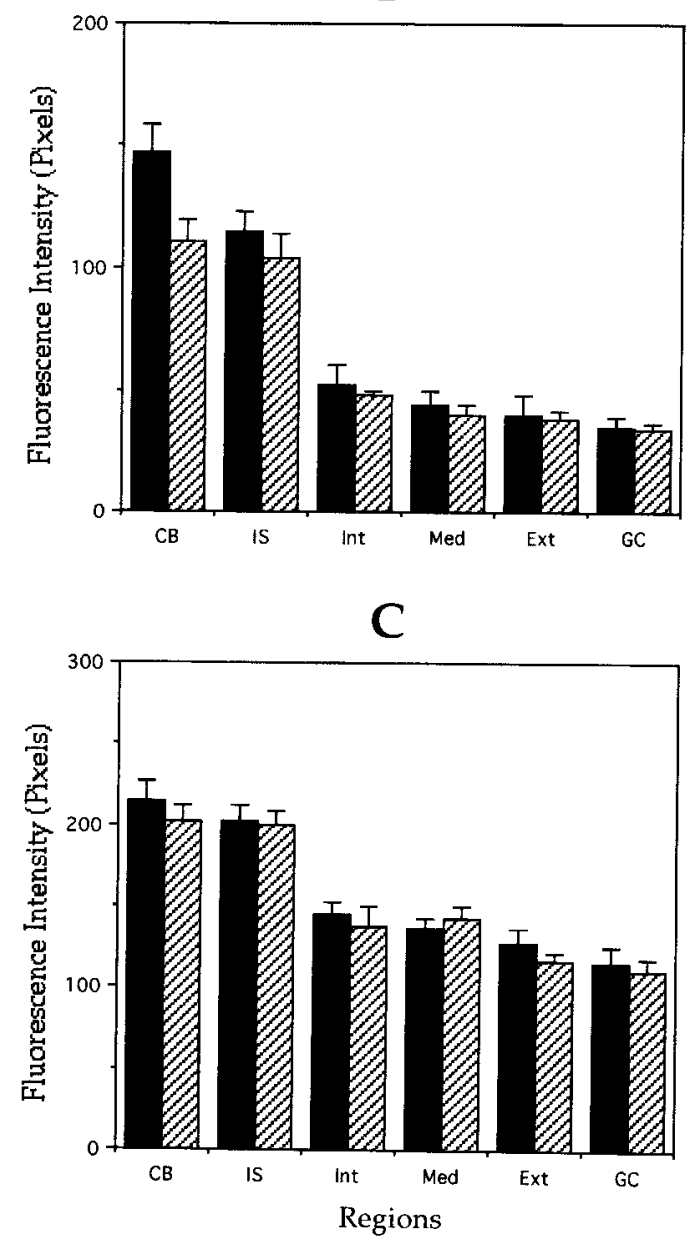

Figure 4. Quantitation of microtubule polymer content of control and MAP1B-deficient neurons. (A and B) Quantitative measurements of $\beta$-tubulin fluorescence intensity in axons (A) and minor

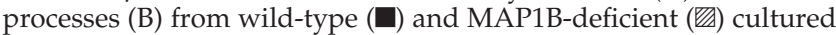
neurons. For these experiments, cells were extracted with detergents before fixation under microtubule-stabilizing conditions (see MATERIALS AND METHODS). Fluorescence intensity measure- with nocodazole declining in amount with a half-life of 5-10 min. For this experiment nocodazole was added to the medium to $5 \mu \mathrm{g} / \mathrm{ml}$ and incubated for up to $60 \mathrm{~min}$. The results obtained show that nocodazole produces a significant decrease in the tyr-microtubule staining of both WT and MAP1B-deficient neurons. Comparisons of tyr-fluorescence intensity within axonal tips of untreated and nocodazoletreated WT neurons revealed that after a 5-min nocodazole pulse, the tyr-tubulin polymer decreases more than $75 \%$; in contrast, a much smaller decrease $(30 \%)$ was detected in equivalent regions of MAP1B-deficient neurons (Figure 6, A and $B$ ). This result suggests that the population of newly stabilized microtubules is relatively larger in MAP1B-deficient neurons than in WT ones, a finding that is consistent with the increase in detyr-microtubule labeling detected in the mutant neurons.

As another test of the consequences of MAP1B suppression on microtubule dynamics, we examined microtubule regrowth during recovery from treatment with nocodazole (30 $\mathrm{min})$. To examine regrowth the cultures were rinsed with fresh medium to remove nocodazole, and incubated for a further 5- to 60-min period. In controls neurons, recovery was evident as the appearance of segments of tyr-tubulin staining in the cell body and at neuritic tips, as soon as $5 \mathrm{~min}$ after the release from nocodazole. In contrast, MAP1B-deficient neurons have an extremely slow microtubule recovery; thus, even $60 \mathrm{~min}$ after nocodazole release, only a slight increase in tyr-microtubule staining was detected in axonal tips of neurons from the MAP1B mutant mice (Figure 6C).

\section{Functional Redundancy in Neurons from MAP1B- deficient Mice}

It has been suggested that a functional redundancy might exist among MAPs (Harada et al., 1994; DiTella et al., 1996; Takei et al., 2000). Therefore, we looked for the existence of such a compensatory mechanism with the use of quantitative fluorescence to measure changes in the relative levels of MAP2 and tau, proteins that have been directly implicated in the regulation of microtubule dynamics and process outgrowth in developing neurons (Cáceres and Kosik, 1990, 1992; Harada et al., 1994). No significant changes in the fluorescence intensity or distribution of MAP2 or tau immunostaining were detected between WT and MAP1B-deficient neurons when cells were fixed before detergent extraction (Figure 7, A and B). In contrast, in "cytoskeletal preparations" a significant increase in MAP2 immunostaining was detected in axons of MAP1B-deficient neurons. However, because these axons are significantly shorter than the ones of WT neurons, the possibility exists that this phenomenon

ments were performed in the cell body $(\mathrm{CB})$ and along neurites. Within neurites measurements were performed in the initial segment (IS), inner segment (Int), middle segment (Med), external segment (Ext), and growth cones (GC) of axons and minor processes. Note that $\beta$-tubulin fluorescence intensity is dramatically reduced in the external axonal segment and in axonal growth cones (C). Equivalent measurements to those shown in A, but from cells extracted with detergents after fixation. Note that there are no significant differences in fluorescence intensity between WT and MAP1B-deficient neurons. A total of 75 cells was analyzed for each experimental condition. Each value represents the mean \pm SEM. 

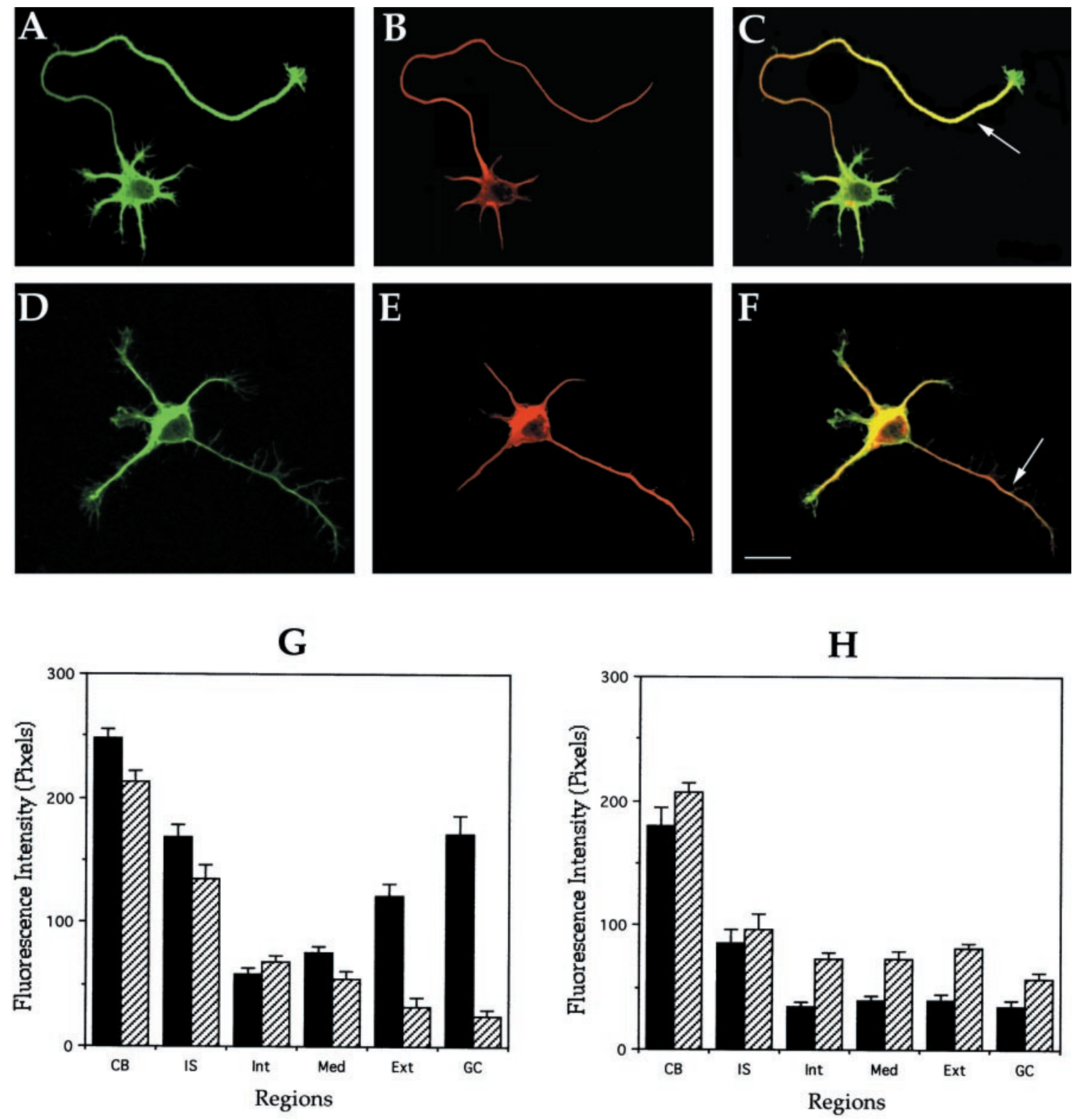

Figure 5. Distribution of stable and dynamic microtubules in control and MAP1B-deficient neurons. (A and B) Confocal fluorescence images showing the distribution of tyrosinated (green) and detyrosinated (red) $\alpha$-tubulin in a cultured hippocampal pyramidal neuron from a wild-type animal. (C) Red-green overlay of the images shown previously. Note that within the axon tyr-tubulin staining predominates at the distal segment (arrow) and growth cone. (D and E) Confocal fluorescence images showing the distribution of tyrosinated (green) and detyrosinated (red) $\alpha$-tubulin in a cultured hippocampal pyramidal neuron from a MAP1B-deficient mice. (F) Red-green overlay of the images shown previously. Note that although the tyr-immunolabeling decreases along the axon, the one corresponding to detyr-tubulin increases (arrow). For this experiment, cells were fixed with detergents under microtubule-stabilizing conditions before fixation. Bar, $10 \mu \mathrm{m}$.

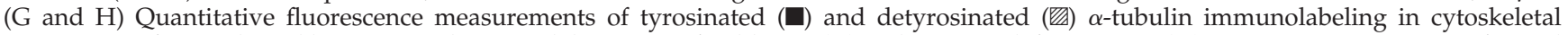
preparations from cultured hippocampal pyramidal neurons of wild-type $(\mathrm{G})$ and MAP1B-deficient mice $(\mathrm{H})$. Measurements were performed in equivalent regions to those described in Figure 4. A total of 75 cells was analyzed for each experimental condition.

reflects a lower degree of maturation (Cáceres et al., 1986) rather than a compensatory mechanism. To distinguish between these possibilities, a comparison of MAP2 immunofluorescence was performed in WT and MAP1B-deficient neurons having axons of equivalent length. Even under this condition we detected significantly more MAP2 immunostaining in ax- ons of mutant neurons than in WT ones (Figure 7, C-F). Tau immunofluorescence associated with the axonal cytoskeleton was almost identical in WT and MAP1B-deficient neurons. Because axons of mutant neurons contain less tubulin polymer than WT ones, these results suggest that tau also increases its association with axonal microtubules in the absence of MAP1B. 


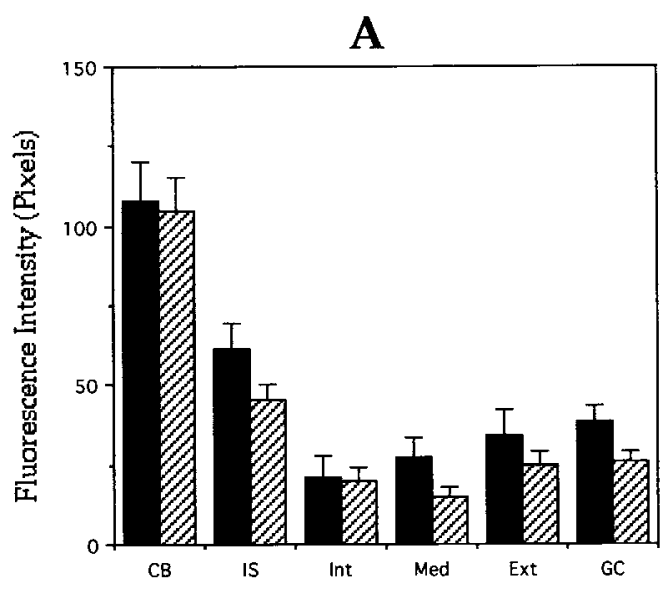

B
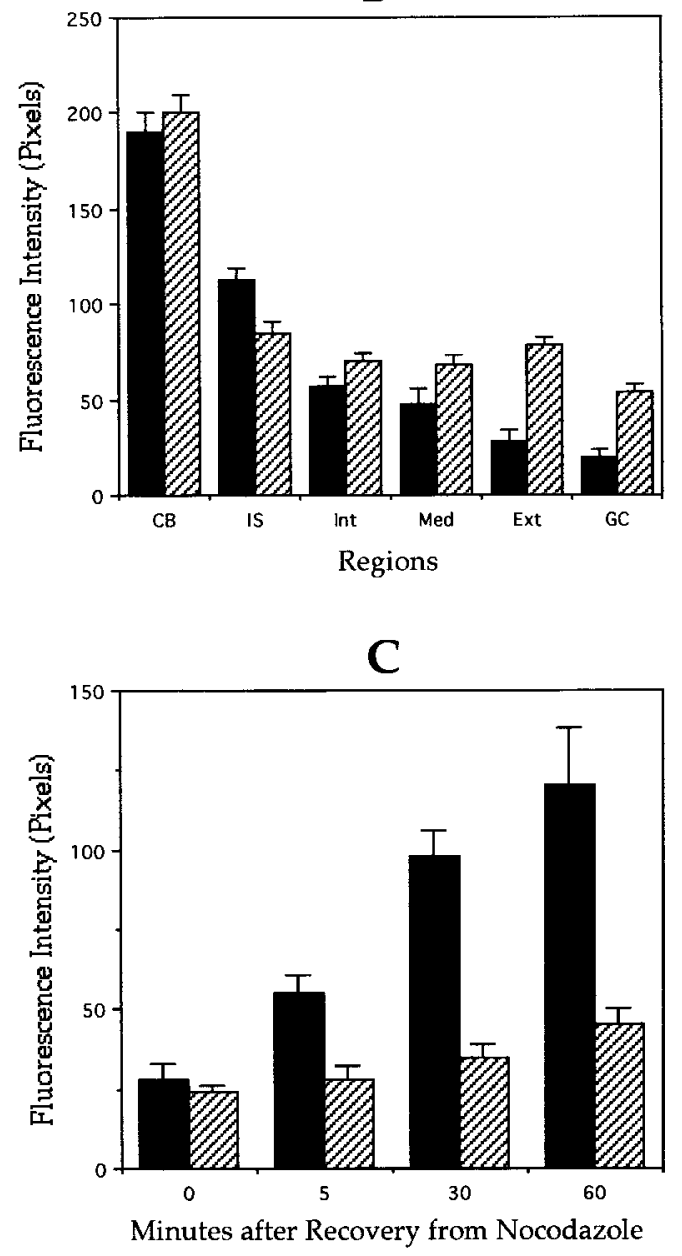

Figure 6. Quantitation of stable and dynamic microtubules in control and MAP1B-deficient neurons. Quantitative fluorescence measurements of tyrosinated (A) and detyrosinated (B) $\alpha$-tubulin immunolabeling in cytoskeletal preparations from wild-type (घ) or MAP1B-deficient (ש) cultured hippocampal pyramidal neurons treated with nocodazole for $5 \mathrm{~min}$. Measurements were performed in equivalent regions to those described in Figure 4. (C) Quantitative

\section{DISCUSSION}

The present results, based on the analysis of cultured neurons obtained from a hypomorphous mouse line having a decrease of $>95 \%$ in MAP1B protein levels (Gonzalez-Billault et al., 2000), provide the first direct experimental evidence suggesting that MAP1B has a crucial participation in axon formation by promoting microtubule assembly and dynamics. Thus, cultured hippocampal pyramidal neurons from MAP1B-deficient mice display a selective delay in axonal outgrowth and also a reduced rate of elongation. This phenotype is paralleled by a significant decrease in the content of axonal microtubules, and particularly of the more dynamic polymer containing tyr-tubulin. Although this phenomenon was observed throughout the entire extent of axons, it was dramatic in the distal axonal segment, as well as in growth cones, where the more recently assembled polymer predominates (Brown et al., 1992; Baas et al., 1993; Li and Black, 1996). Additional evidence, supporting a role for MAP1B in the promotion of microtubule assembly, came from the analysis of microtubule regrowth during recovery from treatment with nocodazole. Whereas in WT neurons recovery was evident as the appearance of segments of tyr-tubulin staining in the cell body and at axonal tips as soon as $5 \mathrm{~min}$ after the release from nocodazole, in MAP1Bdeficient neurons this phenomenon was severely impaired.

Regarding these observations, it is worth noting that the association of MAP1B with axonal microtubules precisely mimics the distribution of newly assembled polymer. In developing neurons MAP1B preferentially binds to microtubules located in the distal axon and the growth cone region (Black et al., 1994; DiTella et al., 1996). An increase in MAP-1B phosphorylation occurs during brain development; more importantly, phosphorylation of MAP1B in "prolinedirected" serine-threonine residues precisely parallels axon formation in cultured neurons and promotes MAP1B binding to microtubules at the distal axonal end (DiTella et al., 1996). Interestingly, two proline-directed protein kinases, namely, cyclin-dependent kinase 5 (Cdk5) and glycogen synthase kinase $3 \beta$ (GSK3 $\beta$ ), which are highly enriched at the distal tip of growing axons and phosphorylate MAP1B, are also required for axonal elongation (Nikolic et al., 1996; Pigino et al., 1997; Paglini et al., 1998a; Lucas et al., 1998).

A question that now arises concerns the relationship between MAP1B-promoted microtubule assembly and stability. The significance of stable microtubules that serve as templates for nucleating tubulin assembly along axonal shafts and at axonal tips is now well established (Baas et al., 1993; Li and Black, 1996). These stable microtubules, which are poor in tyr-tubulin, resistant to nocodazole, and with a half-life of several hours, provide a structural basis for the active tubulin assembly that occurs in growing axons. According to this model, a decrease in stable microtubules would decrease assembly. Because nonneuronal cells over-

fluorescence measurements of tyrosinated $\alpha$-tubulin immunolabeling in cytoskeletal preparations from wild-type ( $\mathbf{\square}$ ) or MAP1Bdeficient (ש) cultured hippocampal pyramidal neurons during recovery from nocodazole. For this experiment cultured neurons were treated with nocodazole for $30 \mathrm{~min}$. Fluorescence intensity measurements were performed at the distal axonal segment. A total of 75 cells was analyzed for each experimental condition. 

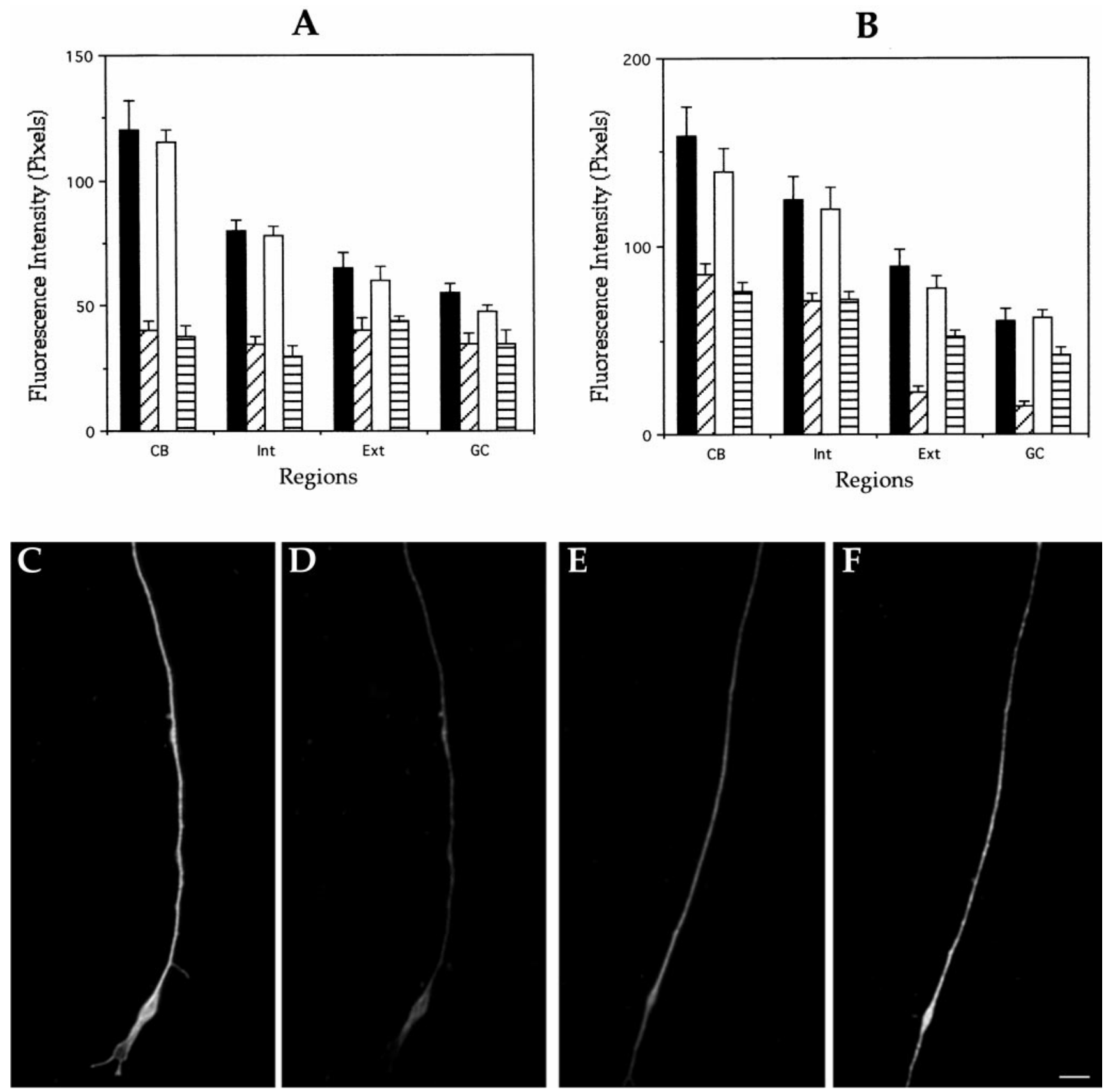

Figure 7. Functional redundancy analysis of control and MAP1B-deficient neurons. Quantitative fluorescence measurements of tau (A) and MAP2 (B) immunolabeling from cultured hippocampal pyramidal neurons of wild-type or MAP1B-deficient neurons. Measurements were performed in cells fixed prior or after detergent extraction performed under microtubule-stabilizing conditions. Regions are equivalent to those of previous figures. Groups: $\mathbf{\square}$, wild-type neurons fixed before extraction; $\mathbb{Z}$, wild-type neurons fixed after extraction; $\square$, MAP1Bdeficient neurons fixed before extraction; and 冒, MAP1B-deficient neurons fixed after extraction. A total of 75 cells was analyzed for each experimental condition. (C-F) Double fluorescence micrographs of cytoskeletal preparations showing $\beta$-tubulin (C and E) and MAP2 (D and F) immunolabeling at the distal axonal third of wild-type ( $C$ and $D)$ or MAP1B-deficient (E and F) neurons. Both axons were of equivalent length. Bar, $5 \mu \mathrm{m}$.

expressing MAPs, including MAP1B heavy and/or light chains, display enhanced microtubule bundling and stability (Takemura et al., 1992; Vandecandelaere et al., 1996; Togel et al., 1998), the possibility exists of MAP1B having a similar role in developing neurons. However, the present results do not favor the view of MAP1B acting as a microtubule-stabilizing factor; they rather suggest that MAP1B acts as a factor limiting microtubule stabilization. Supporting this, we 
showed that axons from MAP1B-deficient neurons have a significant increase in the relative amount of detyr-microtubules and also a higher proportion of recently stabilized polymer. Interestingly, inhibition of GSK3 $\beta$ activity with lithium, which reduces MAP1B phosphorylation and binding to growth cone microtubules (Lucas et al., 1998; Goold et al., 1999), results in a dramatic increase in the number of stable detyr-microtubules in the distal axonal segment of cultured neurons (Goold et al., 1999). Conversely, COS cells transfected with both MAP1B and GSK3 $\beta$ express high levels of phosphorylated MAP1B associated with tyr-microtubules, and a decrease in detyr-tubulin polymer (Goold et al., 1999). Taken together, these observations suggest that, in addition to promoting microtubule assembly, another major function of MAP1B in developing neurons involves maintaining a dynamic microtubule polymer in regions of active growth.

One possible mechanism underlying such a function may involve MAP1B competing with other MAPs for microtubule binding sites. For example, the association of MAP1B with microtubules may result in decreased binding of other MAPs, which otherwise might contribute to microtubule stabilization. Supporting such a proposal, we showed that MAP2 and tau, which are potent microtubule-stabilizing factors (Kanai et al., 1992; Takemura et al., 1992; LeClerk et al., 1993) significantly increase their association with axonal microtubules in MAP1B-deficient neurons.

Regardless of the precise mechanism, MAP1B participation in the maintenance of a dynamic microtubule polymer may be important for allowing interactions between microtubules and actin filaments during growth cone advance and axon extension. Thus, recent studies have shown that dynamic microtubules positively regulate actin dynamics during cell motility (Waterman-Storer and Salmon, 1999). For example, the stabilization of microtubule assembly/disassembly without substantial microtubule polymer loss or disorganization stops cell migration (Liao et al., 1995; Tanaka and Kirschner, 1995; Mikhailov and Gundersen, 1998). Besides, in migrating cells lamellipodial protrusion is highly correlated with the presence of dynamic microtubules near the base of lamellipodia; in contrast, a smaller lamellipodial area and slower movement are associated with microtubules spending more time in pause (Mikhailov and Gundersen, 1998). Moreover, the growth of microtubules in fibroblasts leads to activation of small GTPases such as rac1, which in turn results in actin polymerization and protrusion of the leading lamellipodia (Waterman-Storer and Salmon, 1999; Waterman-Storer et al., 1999). Similar mechanisms appear to operate in neurons because growing microtubules also activate site-directed F-actin assembly in nerve growth cones (Rochlin et al., 1999). Interestingly, immunoelectron microscopy has revealed that microtubules penetrating into the growth cone peripheral domain contain MAP1B and interact with actin filament bundles (Bush et al., 1996; Gordon-Weeks and Fischer, 2000). Therefore, by favoring the existence of dynamic microtubules within growth cones, MAP1B may contribute to the promotion of lamellipodial spreading, an event required for neuronal polarization (Bradke and Dotti, 1997, 1999; Paglini et al., 1998b). In favor of this idea, we observed a significant reduction in the size of the lamellipodial veil and a decrease in the number of actin ribs in axonal growth cones of MAP1B-deficient neurons.
Our results also raise the possibility of MAP1B having a more direct involvement in the regulation of actin organization. Previous studies have shown that MAP1B, and in particular the MAP1B light chain, can bind to actin filaments in vitro (Pedrotti and Islam, 1996), and to stress fibers in vivo (Togel et al., 1998). In addition, it was found that the $\mathrm{COOH}$ terminus of the light chain can efficiently target the $\mathrm{NH}_{2}$ terminus of MAP1B heavy chain to stress fibers (Togel et al., 1998). In this study, we have extended these observations by showing that in MAP1B mutant neurons actin-based protrusive activity is up-regulated and mislocalized. Thus, one striking morphological alteration in MAP1B-deficient neurons is the appearance of numerous short filopodial extensions and growth cone-like structures around the cell body and along neurites. This phenomenon, which is not restricted to axons, may reflect the lack of dephosphorylated MAP1B in the subcortical cytoskeleton of cell bodies, neurites, and growth cones of developing neurons, where it is normally found (DiTella et al., 1996; Paglini et al., 1998a). In future studies, it will be of considerable interest to explore the mechanisms underlying the alterations in actin organization observed in MAP1B-deficient neurons.

Finally, the present results suggest that during the initial stages of axon formation neither MAP2 nor tau are capable of compensating MAP1B deficiency. Although redundant functions of MAP1B and tau have been reported for cultured cerebellar macroneurons treated with antisense oligonucleotides during the initial establishment of neuronal polarity (DiTella et al., 1996; Paglini et al., 2000), no such an effect was observed in MAP1B mutant hippocampal pyramidal neurons. Recently, a double MAP1B and tau mutant mouse has been generated (Takei et al., 2000). Based on the analysis of the phenotype of these animals, the authors claimed the existence of some synergic effects of the two proteins. It is worth noting that the double mutant animals were obtained by breeding a tau mutant line that overexpresses MAP1A in response to the tau deficiency (Harada et al., 1994) with a MAP1B hypomorphic mutant line (Takei et al., 1997). Therefore, this phenotype may not have an easy interpretation and this must be carefully taken into account.

However, the fact that after several days in culture most MAP1B-deficient neurons extend axons, albeit much shorter than those of WT cells, suggests the existence of some kind of mechanism that partially compensates the lack of MAP1B. Whether this involves MAP2, tau, or other MAPs remains to be established.

\section{ACKNOWLEDGMENTS}

This work was supported by grants from the Spanish Dirección General de Investigación Cientifica y Ténica, Comunidad de Madrid, European Union, and an institutional grant from Formation de Recherche Associee to J.A. It was also supported by grants from Consejo Nacional de Investigaciones Cientificas y Técnicas de Argentina (PICT-PIP 4906), FONCyT (PICT 05-00000-00937 and 99-56179), CONICOR, and a Howard Hughes Medical Institute Grant (HMMI 75197-553201) awarded under the International Research Scholars Program to A.C. An A.E.C.I./I.C.I. predoctoral fellowship was awarded to C.G.-B. The MAP1B mutant mouse was generated with financial support from Amgen and the Max Planck Society in the laboratory of Prof. P. Gruss. 


\section{REFERENCES}

Armas-Portela, R., Parrales, M., Albar, J., Martinez, A., and Avila, J. (1999). Distribution and characteristics of bII tubulin-enriched microtubules in interphase cells. Exp. Cell Res. 248, 372-380.

Baas, P., Ahmad, F., Pienkowski, T., Brown, A., and Black, M. (1993). Sites of microtubule stabilization for the axon. J. Neurosci. 13, 21772185.

Black, M., Slaughter, T., and Fischer, I. (1994). Microtubule-associated protein $1 \mathrm{~B}$ (MAP1B) is concentrated in the distal region of growing axons. J. Neurosci. 14, 857-870.

Bloom, G., Luca, F., and Vallee, R. (1995). Microtubule-associated protein 1B: identification of a major component of the neuronal cytoskeleton. Proc. Natl. Acad. Sci. USA 82, 5404-5408.

Bradke, F., and Dotti, C.G. (1997). Neuronal polarity: vectorial cytoplasmic flow precedes axon formation. Neuron 19, 1175-1186.

Bradke, F., and Dotti, C.G. (1999). The role of local actin instability in axon formation. Science 283, 1931-1934.

Brown, A., Slaughter, T., and Black, M. (1992). Newly assembled microtubules are concentrated in the proximal and distal regions of growing axons. J. Cell Biol. 119, 867-882.

Brugg, B., Reddy, D., and Matus, A. (1993). Attenuation of microtubule-associated protein 1B expression by antisense oligodeoxinucleotides inhibits initiation of neurite outgrowth. Neuroscience 52, 489-496.

Cáceres, A., Banker, G., and Binder, L. (1986). Immunochemical localization of tubulin and microtubule-associated protein 2 during the development of hippocampal neurons in culture. J. Neurosci. 6, $714-722$.

Cáceres, A., and Kosik, K. (1990). Inhibition of neurite polarity by antisense oligonucleotides in primary cerebellar neurons. Nature $343,461-463$.

Cáceres A, Mautino, J., and Kosik K. Suppression of MAP-2 in cultured cerebellar macroneurons inhibits minor neurite formation. Neuron 9, 607-618.

Calvert, R., and Anderton, B. (1985). A microtubule-associated protein MAP1 which is expressed at elevated levels during development of rat cerebellum. EMBO J. 4, 1171-1176.

Chowdhury, K., Bonaldo, P., Torres, M., Stoykova, A., and Gruss, P. (1997). Evidence for the stochastic integration of gene trap vectors into the mouse germline. Nuclei Acids Res. 25, 1531-1536.

Craig, A., and Banker, G. (1994). Neuronal polarity. Annu. Rev. Neurosci. 17, 267-310.

DiTella, M., Feiguin, F., Carri, N., Kosik, K., and Cáceres, A. (1996). $\mathrm{MAP} 1 \mathrm{~b} / \mathrm{tau}$ functional redundancy during laminin-enhanced axonal growth. J. Cell Sci. 109, 467-477.

Dotti, C., Sullivan, C., and Banker, G. (1998). The establishment of polarity in hippocampal neurons in culture. J. Neurosci. 8, 14541468.

Drubin, D., Feinstein, S., Shooter, E., and Kirschner, M. (1985). Nerve growth factor induced neurite outgrowth in PC12 cells involves the coordinate induction of microtubule assembly and assembly-promoting factors. J. Cell Biol. 101, 1790-1807.

Edelman, W., Zervas, M., Costello, P., Roback, L., Fischer, I., Hammarback, J., Cowan, N., Davies, P., Wainer, B., and Kucherlapati, R. (1996). Neuronal abnormalities in microtubule-associated protein 1B mutant mice. Proc. Natl. Acad. Sci. USA 93, 1270-1275.

Ferreira, A., Busciglio, J., and Cáceres, A. (1989). Microtubule formation and neurite growth in cerebellar macroneurons which develop in vitro: evidence for the involvement of the microtubule- associated proteins MAP1a, HMW-MAP2 and Tau. Dev. Brain Res. $49,215-228$.

Ferreira, A., and Cáceres, A. (1989). The expression of acetylated microtubules during axonal and dendritic growth in cerebellar macroneurons which develop in vitro. Dev. Brain Res. 49, 205-213.

Fischer, I., and Romano-Clarke, G. (1991). Association of microtubule associated protein (MAP1B) with growing axons in cultured hippocampal neurons. Mol. Cell. Neurosci. 2, 39-51.

Garner, C., Garner, A., Huber, G., Kozak, C., and Matus, A. (1990). Molecular cloning of microtubule-associated protein 5 (MAP1B): identification of distinct genes and their differential expression in developing brain. J. Neurochem. 55, 146-154.

Gonzalez-Billault, C., Demandt, E., Wandosell, F., Torres, M., Bonaldo, P., Stoykova, A., Chowdhury, K., Gruss, P., Avila, J., and Sanchez, M. (2000). Perinatal lethality of microtubule-associated protein $1 \mathrm{~B}$ deficient mice expressing alternative isoforms of the protein at low levels. Mol. Cell. Neurosci. 16, 408-421.

Goold, R., Owen, R., and Gordon-Weeks, P. (1999). (1999). Glycogen synthase kinase 3 beta phosphorylation of microtubule-associated protein $1 \mathrm{~B}$ regulates the stability of microtubules in growth cones. J. Cell Sci. 112, 3373-3384.

Gordon-Weeks, P., and Fischer, I. (2000). MAP1B expression, and microtubule stability in growing, and regenerating axons. Microsc. Res. Tech. 48, 63-74.

Harada, A., Oguchi, K., Okabe, S., Kuno, J., Terada, S., Ohshima, T., Sato-Yoshitake, R., Takei, Y., Noda, T., and Hirokawa, N. (1994). Altered microtubule organization in small caliber axons of mice lacking tau protein. Nature 369, 488-491.

Hummel, T., Krukkert, K., Roos, J., Davis, G., and Klambt, C. (2000). Drosophila Futsch/22C10 is a MAP1B-like protein required for dendritic, and axonal development. Neuron 26, 357-370.

Kanai, Y., Chen, J., and Hirokawa, N. (1992). Microtubule bundling by tau proteins in vivo: analysis of functional domains. EMBO J. 11, 3953-3961.

LeClerk, N., Kosik, K., Cowan, N., Pienkowski, T., and Baas, P. (1993). Process formation in SF9 cells induced by the expression of a microtubule-associated protein 2C-like construct. Proc. Natl. Acad. Sci. USA 90, 6223-6227.

Lein, P., Banker, G., and Higgins, D. (1992). Laminin selectively enhances axonal growth and accelerates the development of polarity by hippocampal neurons in culture. Dev. Brain Res. 69, 191-197.

Li, Y., and Black. M. (1996). Microtubule assembly and turnover in growing axons. J. Neurosci. 16, 531-544.

Liao, G., Nagasaki, T., and Gundersen, G. (1995). Low concentrations of nocodazole interfere with fibroblast locomotion without significantly affecting microtubule level: implications for the role of dynamic microtubules in cell locomotion. J. Cell Sci. 108, 3473-3485.

Lochter, A., and Schachner, M. (1993). Tenascin and extracellular matrix glycoproteins: from promotion to polarization of neurite growth in vitro. J. Neurosci. 13, 3986-4000.

Lucas, F., Goold, R., Gordon-Weeks, P., and Salinas, P. (1998). Inhibition of GSK3 beta leading to the loss of phosphorylated MAP1B is an early event in axonal remodeling induced by WNT-7a or lithium. J. Cell Sci. 111, 1351-1361.

Maccioni, R., and Cambiazo, V. (1995). Role of microtubule-associated proteins in the control of microtubule assembly. Physiol. Rev. $75,835-964$

Mack, T., Koester, M., and Pollerberg, G. (2000). The microtubuleassociated protein MAP1B is involved in local stabilization of turning growth cones. Mol. Cell. Neurosci. 15, 51-65. 
Meixner, A., Haverkamo, S., Wassle, H., Fuhrer, S., Thalhammer, J., Kropf, N., Bitner, R., Lassmann, H., Wiche, G., and Propst, F. (2000). MAP1B is required for axon guidance, and is involved in the development of the central, and peripheral nervous system. J. Cell Biol. 151, 1168-1178.

Mikhailov, A., and Gundersen, G. (1998). Relationship between microtubule dynamics and lamellipodium formation revealed by direct imaging of microtubules in cells treated with nocodazole or taxol. Cell Motil. Cytoskeleton 41, 325-340.

Mitchison, T., and Kirschner, M. (1989). Cytoskeletal dynamics and nerve growth. Neuron 1, 761-772.

Nikolic, M., Dudek, H., Kwon, Y., Ramos, Y., and Tsai, L.H. (1996). The cdk5/p35 kinase is essential for neurite outgrowth during neuronal differentiation. Genes Dev. 10, 816-825.

Paglini, G., Kunda, P., Quiroga, S., Kosik, K., and Cáceres, A. (1998b). Suppression of radixin and moesin alters growth cone morphology, motility, and process formation in primary cultured neurons. J. Cell Biol. 143, 445-455.

Paglini, G., Peris, L., Mascotti, F., Quiroga, S., and Cáceres, A. (2000). Tau protein function during axonal formation. Neurochem. Res. 25, 37-42.

Paglini, G., Pigino, G., Kunda, P., Morfini, G., Maccioni, R., Quiroga, S., Ferreira, A., and Cáceres, A. (1998a). Evidence for the involvement of the neuron-specific cdk5 activator p35 during lamininenhanced axonal growth. J. Neurosci. 18, 9858-9869.

Pedrotti, B., and Islam, K. (1996). Dephosphorylated but not phosphorylated microtubule-associated protein MAP1B binds to microfilaments. FEBS Lett. 388, 131-133.

Rochlin, M., Dailey, M., and Bridgman, P. (1999). Polymerizing microtubules activate site-directed F-actin assembly in nerve growth cones. Mol. Biol. Cell 10, 2309-2327.

Roos, J., Hummel, T., Ng, N., Klambt, C., and Davis, G. (2000). Drosophila Futsch regulates microtubule organization, and is necessary for synaptic growth. Neuron 26, 371-382.
Sanchez-Martin, C., Diaz-Nido, J., and Avila, J. (1998). (1998). Regulation of a site-specific phosphorylation of the microtubule-associated protein 2 during the development of cultured neurons. Neuroscience $87,861-870$.

Takei, Y., Kondo, S., Harada, A., Inomata, S., Noda, T., and Hirokawa, N. (1997). Delayed development of nervous system in mice homozygous for disrupted microtubule-associated protein 1B (MAP1B) gene. J. Cell Biol. 137, 1615-1626.

Takei, Y., Teng, J., Harada, A., and Hirokawa, N. (2000). Defects in axonal elongation, and neuronal migration in mice with disrupted tau, and map1b genes. J. Cell Biol. 150, 989-1000.

Takemura, R., Okabe, T., Umeyama, Y., Kanai, Y., Cowan, N., and Hirokawa, N. (1992). Increased microtubule stability and alpha tubulin acetylation in cells transfected with microtubule associated protein MAP1B, MAP2 or tau. J. Cell Sci. 103, 953-964.

Tanaka, E., and Kirschner, M. (1995). The role of microtubule dynamics in growth cone motility and axonal growth. J. Cell Biol. 128, 139-155.

Togel, M., Wiche, G., and Propst, F. (1998). Novel features of the light chain of microtubule-associated protein MAP1B: microtubulestabilization, self interaction, actin filament binding, and regulation by the heavy chain. J. Cell Biol. 143, 695-702.

Ulloa, L., Avila, J., and Diaz-Nido, J. (1993). Heterogeneity in the phosphorylation of microtubule-associated protein MAP1B during rat brain development. J. Neurochem. 61, 961-972.

Vandecandelaere, A., Pedrotti, B., Utton, M., Calvert, R., and Bayley, P. (1996). Differences in the regulation of microtubule dynamics by microtubule-associated protein MAP1B and MAP2. Cell Motil. Cytoskeleton 35, 134-146.

Waterman-Storer, C., and Salmon, E. (1999). Positive feed-back interactions between microtubules and actin dynamics during cell motility. Curr. Opin. Cell Biol. 11, 61-67.

Waterman-Storer, C., Worthylake, R., Liu, B., Burridge, K., and Salmon, E. (1999). Microtubule growth activates Rac1 to promote lamellipodial protrusion in fibroblasts. Nat. Cell Biol. 1, 45-50. 